\title{
Multiple roles of extracellular fibroblast growth factors in lung cancer cells
}

\author{
TAKAHIRO SUZUKI $^{1 *}$, HIROYUKI YASUDA $^{2}$, KOJI FUNAISHI $^{*}{ }^{*}$, DAISUKE ARAI $^{2}$, KOTA ISHIOKA $^{2}$, \\ KEIKO OHGINO $^{2}$, TETSUO TANI ${ }^{2}$, JUNKO HAMAMOTO ${ }^{2}$, AYANO OHASHI ${ }^{2}$, \\ KATSUHIKO NAOKI ${ }^{3}$, TOMOKO BETSUYAKU ${ }^{2}$ and KENZO SOEJIMA ${ }^{2}$ \\ ${ }^{1}$ Keio University School of Medicine; ${ }^{2}$ Division of Pulmonary Medicine, \\ Department of Medicine, Keio University School of Medicine; \\ ${ }^{3}$ Keio Cancer Center, Keio University Hospital, Shinjuku-ku, Tokyo 160-8582, Japan
}

Received August 9, 2014; Accepted September 18, 2014

DOI: $10.3892 /$ ijo.2014.2718

\begin{abstract}
Cancer cells are surrounded by the extracellular fluid, matrix, and stroma cells. Little is known about how extracellular components such as growth factor ligands affect the biology of lung cancer cells. The objective of this study was to determine whether extracellular fibroblast growth factors (FGFs) can affect the biology of lung cancer cells and to understand how extracellular FGFs affect the biology of lung cancer cells, including non-small cell lung cancer (NSCLC) and small cell lung cancer (SCLC) cells. Out of the 23 reported FGFs, we focused on FGF2, FGF9 and FGF10. We examined the effect of FGFs on proliferation, treatment sensitivity, and apoptosis of NSCLC (PC9) and SCLC (H69, $\mathrm{H} 82$ and H146) cells in vitro. To determine which FGF was the most clinically relevant, we also examined FGF2 and FGF9 concentrations in the serum of patients with lung cancer. We found that extracellular FGFs can affect proliferation, treatment sensitivity, and apoptosis of lung cancer cells in a cell-specific manner. Our results indicate that extracellular FGFs affect the biology of lung cancer cells through multiple functions.
\end{abstract}

\section{Introduction}

Lung cancer is the leading cause of cancer-related death worldwide (1). In general, lung cancer is histologically classified as non-small cell lung cancer (NSCLC) and small cell lung cancer (SCLC), which account for $\sim 85$ and $15 \%$ of all

Correspondence to: Dr Hiroyuki Yasuda, Division of Pulmonary Medicine, Department of Medicine, Keio University School of Medicine, 35 Shinanomachi, Shinjuku-ku, Tokyo 160-8582, Japan E-mail: hiroyukiyasuda@a8.keio.jp

${ }^{*}$ Contributed equally

Key words: non-small cell lung cancer, small cell lung cancer, fibroblast growth factors lung malignancies, respectively. To overcome this deadly disease, considerable efforts have been made to identify the molecular mechanisms of lung cancer initiation and progression. Especially, the recent advance in comprehensive genome analysis tools revolutionized our understanding of lung cancer molecular biology (2-6). These advances led to the identification of several critical genes for lung cancer. These include the epidermal growth factor receptor $(E G F R)$, Kirsten rat sarcoma viral oncogene homolog $(K-R A S)$, anaplastic lymphoma kinase $(A L K)$, and fibroblast growth factor receptor (FGFR) (6-12). Somatic alterations of these genes, including gene mutations or gene rearrangements, induce protein activation, which subsequently induces the activation of downstream pathways critical for lung cancer cell survival and proliferation. These pathways include the phosphatidylinositol-3-kinase/protein kinase B (PI3K/AKT) and the extracellular signal-regulated kinase (ERK)/mitogen-activated protein kinase (MAPK) pathways. The effects of receptor activation on these downstream pathways have been clearly demonstrated in vitro $(13,14)$. Cancer cells are surrounded by the extracellular matrix, extracellular fluid, and stroma cells. The aforementioned cell-autonomous alterations of the receptors can affect cancer cell biology, but we also speculate that non-cellautonomous alterations such as ligand concentration in the microenvironment can affect the biology of lung cancer cells.

Recently, fibroblast growth factors (FGFs) and their receptors, FGFRs, attracted the attention of lung cancer researchers, because FGFR somatic activating mutations and gene amplifications have been repeatedly reported in lung cancer $(5,15)$. Moreover, some FGFR inhibitors are reported to be effective in NSCLC (16) and SCLC (17). FGFR activation induces the activation of the downstream PI3K/AKT and MAPK pathways $(18,19)$, promoting lung cancer cell proliferation and inhibiting lung cancer cell apoptosis. FGFs are known to induce the activation of FGFRs, which subsequently induce downstream proteins activation. The FGF family comprises 23 FGFs, and the roles of some FGFs, especially FGF2, have been well studied in the cancer field $(20,21)$. Recently Wilson and colleagues reported that receptor tyrosine kinase (RTK)driven cancer cell sensitivity to the corresponding RTK inhibitors was affected by the extracellular ligand concentra- 
tion (22). In this study, they used several ligands, including FGF2 and found that extracellular ligands can reduce cancer cell sensitivity to specific RTK-inhibitors.

However, whether extracellular FGFs affect lung cancer cell functions, such as, proliferation, treatment sensitivity, and apoptosis, remain unclear. Moreover, which FGFs are potent and how extracellular FGFs affect lung cancer cell biology remain unclear.

The objective of this study was to determine whether extracellular FGFs can affect lung cancer cell biology and to understand how extracellular FGFs affect the biology of NSCLC and SCLC. In this study, out of the 23 reported FGFs, we focused on FGF2, FGF9, and FGF10. FGF2 and FGF10 were chosen because they have been studied in some cancers, including lung cancer $(21,23)$. FGF9 was chosen because we have previously reported that patients with NSCLC with high FGF9 expression present a worse prognosis than patients with low FGF9 expression (24). We also reported that lung specific expression of FGF9 induced the formation of lung adenocarcinoma in a genetically engineered mouse model (25). In this study, we used NSCLC (PC9) and SCLC (H69, H82 and H146) cells. We also examined FGF2 and FGF9 concentrations in the serum of patients with lung cancer.

\section{Materials and methods}

Cell culture. PC9 (NSCLC, EGFR exon 19 deletion, EGFRdelE746-A750), H69 (SCLC), H82 (SCLC), and H146 (SCLC) cell lines were used in this study. PC9 was obtained as previously described (26). The other cell lines were purchased from the American Type Culture Collection (ATCC, Manassas, VA, USA). All cell lines were cultured in RPMI-1640 growth medium, supplemented with $10 \%$ fetal bovine serum (FBS) at $37^{\circ} \mathrm{C}$ in a humidified $5 \% \mathrm{CO}_{2}$ incubator.

Reagents. Recombinant human FGF2, FGF9 and FGF10 were purchased from Peprotech (Rocky Hill, NJ, USA). In this study, the FGFs was used at $100 \mathrm{ng} / \mathrm{ml}$ as previously described $(23,27,28)$. Erlotinib was purchased from LC Laboratories (Boston, MA, USA). Docetaxel was purchased from Wako (Osaka, Japan). Etoposide was purchased from Sigma-Aldrich Japan (Tokyo, Japan). Phospho-p44/42 MAPK (MAPK Tyr202/Tyr204) (p-ERK1/2) antibody (no. 3126), total p44/42 MAPK (ERK1/2) antibody (no. 3127), phospho-AKT (Ser473; D9E) (p-AKT) antibody (no. 4060), and total AKT antibody (no. 9272) were purchased from Cell Signaling Technologies (Danvers, MA, USA).

Quantitative RT-PCR. Total RNA was prepared using an RNeasy Mini kit (Qiagen, Hilden, Germany) and the RNA was then reverse transcribed to cDNA using TaqMan Reverse Transcription reagents (Invitrogen, Carlsbad, CA, USA). For quantitative RT-PCR analysis, we used the ABI PRISM 7000 Sequence Detection system (Life Technologies, Carlsbad, CA, USA). Human glyceraldehyde-3-phosphate dehydrogenase (GAPDH) was used for normalization of input cDNA. The probes used in this study were, Hs00915142 for FGFR1, Hs01552926 for FGFR2, Hs00179829 for FGFR3, Hs01106908 for FGFR4, and H99999905 for GAPDH.
MTS proliferation assay. Evaluation of the proliferation was done using an MTS proliferation assay (CellTiter 96 AQueous One Solution Assay kit, Promega, Madison, WI, USA). The MTS proliferation assay was conducted according to the manufacturer's protocol. Briefly, 2.0 $\times 10^{3}$ cells were seeded per well in 96-well plates. The cells were then treated with or without FGF2 (100 ng/ml), FGF9 (100 ng/ml), and FGF10 (100 ng/ml). Control cells were treated with the same concentration of the vehicle, dimethyl sulfoxide (DMSO). Seventy-two hours after treatment, the number of viable cells was measured.

Western blotting. Proteins were extracted using a cell lysis buffer (Cell Signaling Technologies). Protein concentrations were quantified by BCA protein assay (Thermo Scientific, Waltham, MA, USA) and equal amounts of protein were denatured and reduced with sample buffer. After boiling, aliquots of the samples were subjected to electrophoresis. The fractionated proteins were transferred to polyvinylidene difluoride (PVDF) membranes. The membrane was incubated with the diluted primary antibodies followed by incubation with the secondary antibodies. For the protein detection, the membrane was incubated with agitation in LumiGLO reagent and peroxide (Cell Signaling Technologies) and exposed to X-ray film.

Erlotinib sensitivity assay. Erlotinib sensitivity was evaluated using the MTS proliferation assay with or without erlotinib and with or without FGFs. Briefly, $3.0 \times 10^{3}$ cells were seeded per well in 96-well plates. The cells were then treated with erlotinib and with or without FGF2 (100 ng/ml), FGF9 (100 ng/ml), and FGF10 (100 ng/ml). Control cells were treated with the same concentration of DMSO. Seventy-two hours after treatment, the number of viable cells was measured.

Apoptosis assay. PC9 cells were seeded in 6-well plates at 100,000 cells per well for flow cytometric analysis using Gallios (Beckman Coulter, Brea, CA, USA). The cells were then treated with or without erlotinib at $1 \mu \mathrm{mol} / 1$ for $48 \mathrm{~h}$. As a control, cells were treated with the same concentration of DMSO. We analyzed the cell apoptotic status using TACS Annexin V-FITC (R\&D Systems, Minneapolis, MN, USA) according to the manufacturer's protocol.

Human serum sample collection and FGF2 and FGF9 quantification. All human samples were obtained with written informed consent. This study was reviewed and approved by the Institutional Review Board of Keio University School of Medicine. Fifteen patients with lung cancer and 8 patients with other lung disease, including infectious lung disease, interstitial lung disease and chronic obstructive pulmonary disease (COPD) were included in this study. All serum samples were obtained before chemotherapy. FGF2 and FGF9 concentrations were measured using enzyme-linked immunosorbent assay (ELISA). FGF2 and FGF9 ELISA were purchased from R\&D Systems and Ray Biotech Inc. (Norcross, GA, USA), respectively.

Statistical analysis. All p-values are two-sided. Student's t-test was used for comparison in this study. The p-values $<0.05$ were considered statistically significant. 
A

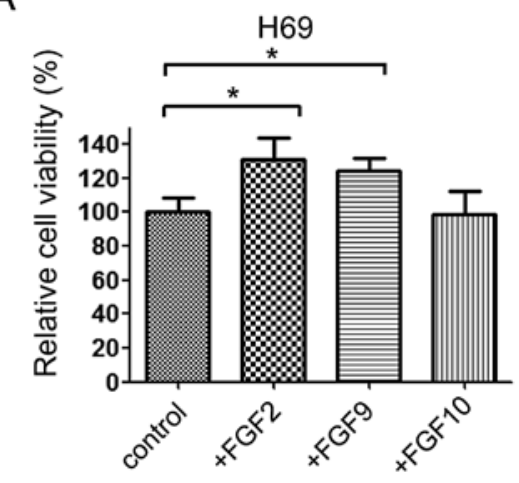

C

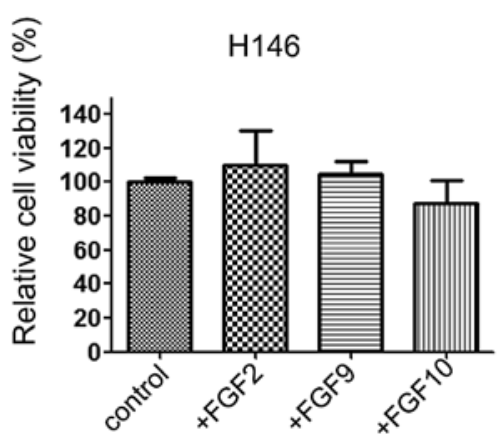

B

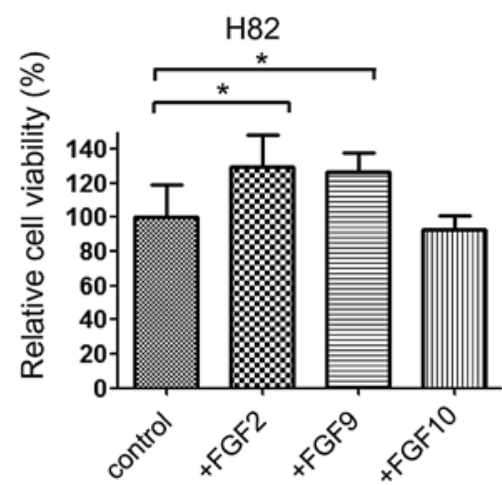

D

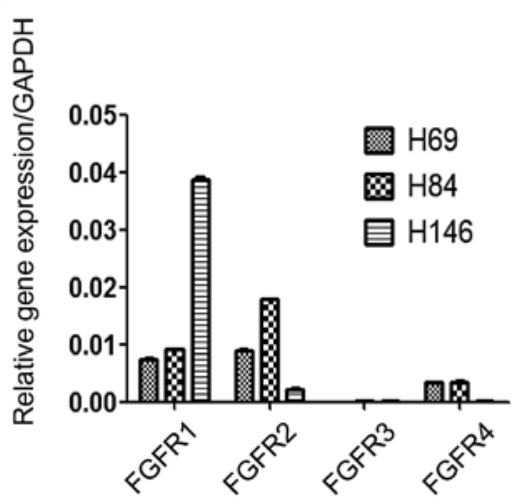

Figure 1. The FGF effects on SCLC cell proliferation. (A) The relative viability of H69 cells in the presence or absence of FGF2, FGF9 and FGF10. (B) The relative viability of $\mathrm{H} 82$ cells in the presence or absence of FGF2, FGF9 and FGF10. (C) The relative viability of H146 cells in the presence or absence of FGF2, FGF9 and FGF10. (D) FGFR1, FGFR2, FGFR3 and FGFR4 relative gene expression in H69, H82, and H146 cells. The data represent mean values $\pm \mathrm{SD}$. ${ }^{*} \mathrm{p}<0.05$.

\section{Results}

FGFs promotes SCLC cell proliferation in a cell-specific manner. First, to determine whether FGFs promoted lung cancer cell proliferation, the lung cancer cells were incubated with or without FGFs. None of the three FGFs promoted NSCLC PC9 cell proliferation. However, FGF2 and FGF9 significantly increased the proliferation of $\mathrm{H} 82$ and $\mathrm{H} 69$ cells, two of the SCLC cell lines. Interestingly, FGFs exerted their effects on lung cancer cell proliferation in a cell-specific manner. In H69 and H82 cells, FGF2 and FGF9 significantly increased the proliferation, while FGF10 did not (Fig. 1A and B). In H146 cells, none of the FGFs affected cell proliferation (Fig. 1C). To investigate this discrepancy, quantitative RT-PCR was performed to determine the expression of FGFRs. We found that FGFR1 expression was observed in all three cell lines, while FGFR3 expression was not. FGFR2 and FGFR4 were preferentially expressed in H69 and H82 cells, which were affected by FGF2 and FGF9 in terms of proliferation (Fig. 1D). Thus, we speculate that FGF2 and FGF9 effects on proliferation are mediated through FGFR2 and FGFR4.

FGFs activate the PI3K/AKT and MAPK pathways. Western blot analysis was performed to understand how FGF signal is transduced to cellular downstream pathways, including the PI3K/AKT and MAPK pathways. To evaluate the activation of the PI3K/AKT and MAPK pathways, the phosphorylated form of AKT (p-AKT) and ERK1/2 (p-ERK1/2) were detected by western blot analysis (Fig. 2). FGF stimulation only modestly
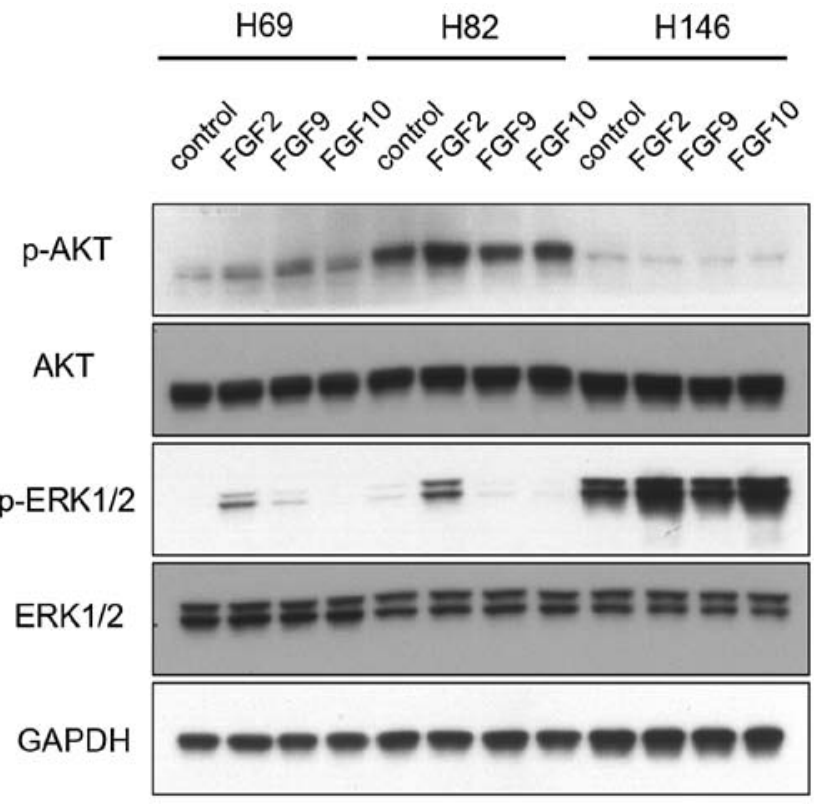

Figure 2. FGF effects on downstream pathways in SCLC cells. Phosphorylation of AKT (p-AKT) and ERK1/2 (p-ERK1/2), total AKT and ERK1/2, and GAPDH were analyzed by western blotting. FGF concentration was $100 \mathrm{ng} / \mathrm{ml}$.

increased the p-AKT signal. In H69 cells, FGF2, FGF9, and FGF10 slightly increased the p-AKT signal. In H82 cells, only FGF2 slightly increased the p-AKT signal. 
A

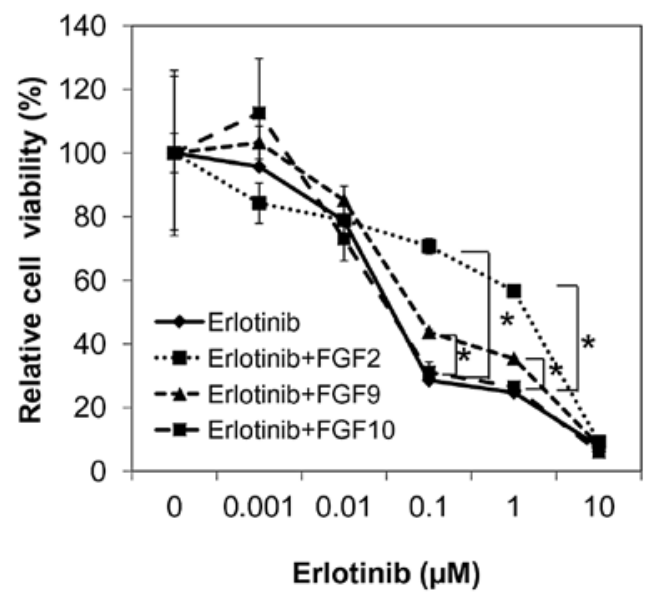

B

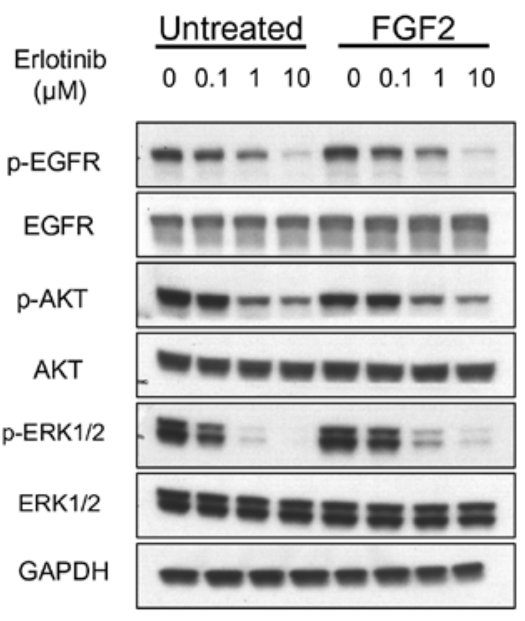

Figure 3. FGF effects on PC9 cell sensitivity to erlotinib. (A) Results of the MTS proliferation assay are shown. PC9 cells were treated with erlotinib and with or without FGFs. PC9 cell relative viability was determined at each erlotinib concentration. The data represent mean values \pm SD. ${ }^{*} \mathrm{p}<0.05$. (B) Phosphorylation of EGFR, AKT, and ERK1/2, total EGFR, AKT, ERK1/2 and GAPDH were analyzed by western blotting.

On the contrary, the effect of FGFs on the activation of the MAPK pathway was significant. FGF2 dramatically increased p-ERK1/2 in H69 and H82 cells. FGF9 increased p-ERK1/2 in H69 cells. However, FGF10 only slightly increased p-ERK1/2 in $\mathrm{H} 146$ cells.

These data indicate that FGF signal is mainly transduced through the MAPK pathway rather than the PI3K/AKT pathway. Of the three FGFs tested, FGF2 was the most potent in activating the MAPK pathway. Although there is some discrepancy between the aforementioned cell proliferation data and the MAPK pathway activation, we speculate that the effect of FGFs on cell proliferation is mediated through the MAPK pathway.

FGFs induce erlotinib resistance in PC9 cells. To investigate whether extracellular FGFs can affect the sensitivity of cancer cells to anticancer drugs, we performed the MTS proliferation assay with or without several anticancer drugs. For SCLC cells, we used etoposide and docetaxel. However, the three FGFs did not affect H69, H82, and H146 sensitivity to these drugs (data not shown).

As previously described, PC9 cells are sensitive to EGFR tyrosine kinase inhibitors, such as gefitinib and erlotinib. In our study, we used erlotinib. Erlotinib alone inhibited PC9 cell proliferation as previously described. FGF2 and FGF9 induced a significant resistance to erlotinib in PC9 cells when compared with erlotinib alone, while FGF10 did not (Fig. 3A). FGF2 was more potent in inducing resistance to erlotinib than FGF9. These data indicate that FGFs, especially FGF2, can affect NSCLC cell sensitivity to erlotinib.

To determine how FGFs, especially FGF2, affected erlotinib sensitivity, western blot analysis was performed to assess the effect on downstream pathways. PC9 cells were treated with erlotinib and with or without FGF2. Erlotinib effectively inhibited the phosphorylation of EGFR irrespective of the presence of FGF2. The effect of FGF2 on p-AKT was modest. However, FGF2 induced an increase in p-ERK1/2 in the presence of 0.1 , 1.0 , and $10 \mu \mathrm{M}$ erlotinib (Fig. 3B). These data indicate that
FGF2 induced erlotinib resistance through activation of the MAPK pathway rather than the PI3K/AKT pathway.

FGFs inhibit erlotinib-induced apoptosis in PC9 cells. To determine whether FGFs affect erlotinib-induced apoptosis in PC9 cells, apoptosis was analyzed using Annexin V-FITC and propidium iodide (PI) double staining and flow cytometry. PC9 cells were incubated with erlotinib and with or without FGFs for $48 \mathrm{~h}$. Erlotinib alone induced early phase apoptosis in $19.5 \%$ of the cells, and late phase apoptosis in $42.61 \%$ of the cells. FGF2, but not FGF9 and FGF10, inhibited erlotinib-induced apoptosis in PC9 cells. FGF2 reduced the proportion of cells in the late phase apoptosis from 42.61 to $15.21 \%$ and increased the proportion of non-apoptotic cells. When compared to erlotinib alone, the proportion of double negative cells, PI-negative and Annexin V-FITC-negative cells, increased from 23.69 to $41.19 \%$ when the cells were treated with erlotinib and FGF2 (Fig. 4). These data indicate that FGF2 affect erlotinib-induced apoptosis in PC9 cells.

FGF2 and FGF9 concentrations in human serum samples. To determine the clinical relevance of extracellular FGFs in patients with lung cancer, we measured FGF serum concentrations in 15 patients with lung cancer and 8 patients with other lung disease. Of the three FGFs tested in this study, FGF2 and FGF9 presented some functional effects on the cancer cell biology, while FGF10 did not. Thus, we focused on FGF2 and FGF9. FGF2 and FGF9 specific ELISAs were used to quantify FGF2 and FGF9 serum concentrations. The clinical characteristics of the patients are presented in Fig. 5A. The mean serum FGF2 concentration was $5.03 \mathrm{pg} / \mathrm{ml}$ in patients with lung cancer, while it was $3.94 \mathrm{pg} / \mathrm{ml}$ in patients with other lung disease (Fig. 5B). Although the concentration was higher in patients with lung cancer than in patients with other lung disease, the difference was not statistically significant $(p=0.58)$. The mean serum FGF9 concentration was less than the detectable range in patients with lung cancer and other lung disease (Fig. 5B). 


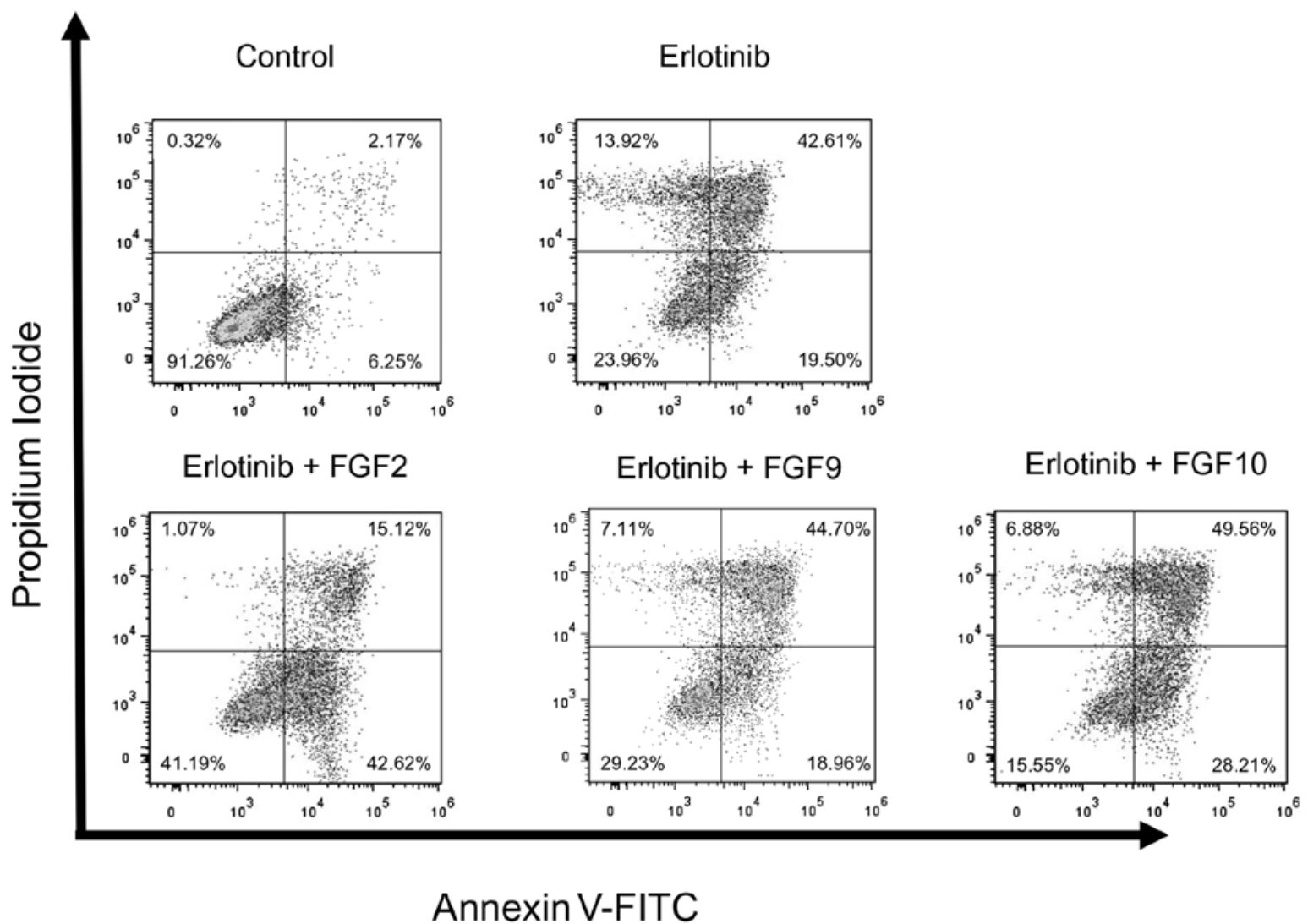

Figure 4. FGF effects on erlotinib-induced apoptosis in PC9 cells. Results of Annexin V-FITC and propidium iodide (PI) staining analysis using flow cytometry are shown. PC9 cells were incubated with erlotinib with or without FGFs for $48 \mathrm{~h}$. The proportion of each quadrant is presented. FGF concentration was $100 \mathrm{ng} / \mathrm{ml}$.

A

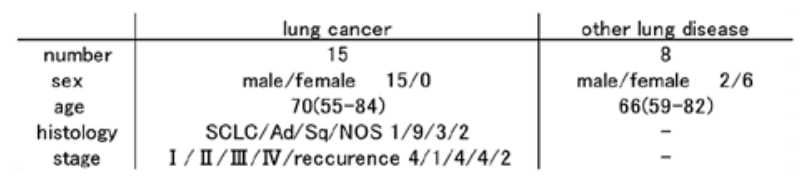

B

mean serum concentration $(\mathrm{pg} / \mathrm{ml})$

\begin{tabular}{r|c|c} 
& lungcancer & other lung disease \\
\hline FGF2 & 5.03 & 3.94 \\
FGF9 & undetectable & undetectable \\
FGF10 & N.A. & N.A.
\end{tabular}

C

FGF2

p-value: 0.58

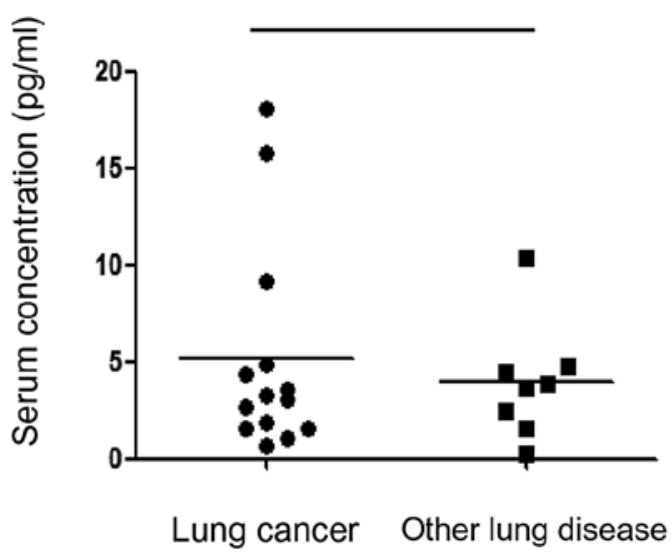

Figure 5. FGF2 and FGF9 concentrations in the patients with lung cancer. (A) Patients clinical characteristics. SCLC, small cell lung cancer. Ad, adenocarcinoma. Sq, squamous cell carcinoma. NOS, not otherwise specified. (B) FGF2 and FGF9 mean serum concentrations. (C) Dot plot analysis of FGF2 concentrations in serum from 15 patients with lung cancer and 8 patients with other lung disease. Each dot indicates the serum concentration of the patient. Bar indicates the mean value of FGF2.

\section{Discussion}

To date, many 'cell autonomous' cancer-specific alterations such as EGFR gene mutation, FGFR gene mutation, or $A L K$ translocation have been characterized. The characterization enabled us to develop molecular targeted therapy for the altered gene itself or its downstream pathways. However, the tumor microenvironment may affect the biology of lung 
cancer cells. Characterization of the effect of extracellular microenvironment on cancer cells may help us to develop new therapies targeting 'non-cell autonomous' alteration of the cancer cell microenvironment. In this study, among the various microenvironment components, we focused on FGF ligands.

The FGF family include 23 proteins, although only 18 are FGFR ligands (19). The FGFs, which function as FGFR ligands, activate FGFR, which, in turn, phosphorylates the FGFR substrate 2 (FRS2) and recruits growth factor receptorbound 2 (GRB2), finally resulting in the activation of the MAPK and PI3K/AKT pathways (18). Of all FGFs, FGF2 is the most studied in the cancer field. However, little is known about the roles of other FGFs. In this study, we attempted to determine which FGF among FGF2, FGF9, and FGF10, was the most potent in promoting lung cancer cell proliferation or in inhibiting lung cancer cell apoptosis.

Using cell line models, we found that FGFs can affect the biology of NSCLC and SCLC cells. We determined that some FGFs can activate the MAPK pathway, promote lung cancer cell proliferation, and change lung cancer cell sensitivity to erlotinib.

Using human serum samples, we found the concentration of FGF2 was higher in patients with lung cancer compared with that in patients with other lung disease, although it was not statistically significant. We cannot determine whether the concentration of FGF2 is higher in patients with lung cancer compared with that in patients with other lung disease, because the human study was incomplete with small patients number and several confounding factors. Moreover, we cannot determine whether serum FGF2 in patients with lung cancer was derived from lung cancer tissue, because FGFs are not specifically secreted from lung cancer cells.

We found that concentration of FGF2 was higher than that of FGF9. The FGF9 serum concentration was below the detectable limit by ELISA assay. Although FGF serum concentrations may not directly reflect FGF concentration in the microenvironment of lung cancer cells, combining the in vitro and human serum results, we speculate that, out of the three FGFs tested, FGF2 is the most potent FGF in promoting proliferation and in inhibiting apoptosis of lung cancer cells. When comparing FGF9 and FGF10, FGF9 is more likely to promote proliferation and inhibit apoptosis than FGF10.

In conclusion, our study demonstrates that FGFs have multiple roles in the biology of lung cancer cells. To thoroughly understand lung cancer cell biology, further studies of not only cell autonomous alterations, but also non-cell autonomous alterations are mandatory. Further studies on the role of extracellular ligands may help us develop novel treatments targeting extracellular ligands.

\section{Acknowledgements}

We thank Mikiko Shibuya for her excellent technical assistance. This study was supported in part by Grants-in-Aid for Scientific Research on Priority Areas from the Ministry of Education, Culture, Sports, Science, and Technology of Japan to K.S. (grant no. 22590870), H.Y. (grant no. 25860656), K.N. (grant no. 23501311), and T.B. (grant no. 23390218).

\section{References}

1. Siegel R, Naishadham D and Jemal A: Cancer statistics, 2013. CA Cancer J Clin 63: 11-30, 2013.

2. Cancer Genome Atlas Research Network: Comprehensive genomic characterization of squamous cell lung cancers. Nature 489: 519-525, 2012.

3. Imielinski M, Berger AH, Hammerman PS, et al: Mapping the hallmarks of lung adenocarcinoma with massively parallel sequencing. Cell 150: 1107-1120, 2012.

4. Wistuba, II, Gazdar AF and Minna JD: Molecular genetics of small cell lung carcinoma. Semin Oncol 28: 3-13, 2001.

5. Peifer M, Fernandez-Cuesta L, Sos ML, et al: Integrative genome analyses identify key somatic driver mutations of small-cell lung cancer. Nat Genet 44: 1104-1110, 2012.

6. Weir BA, Woo MS, Getz G, et al: Characterizing the cancer genome in lung adenocarcinoma. Nature 450: 893-898, 2007.

7. Paez JG, Janne PA, Lee JC, et al: EGFR mutations in lung cancer: correlation with clinical response to gefitinib therapy. Science 304: 1497-1500, 2004.

8. Yasuda H, Kobayashi S and Costa DB: EGFR exon 20 insertion mutations in non-small-cell lung cancer: preclinical data and clinical implications. Lancet Oncol 13: e23-31, 2012.

9. Yasuda $\mathrm{H}$, Park E, Yun $\mathrm{CH}$, et al: Structural, biochemical, and clinical characterization of epidermal growth factor receptor (EGFR) exon 20 insertion mutations in lung cancer. Sci Transl Med 5: 216ra177, 2013.

10. Soda M, Choi YL, Enomoto M, et al: Identification of the transforming EML4-ALK fusion gene in non-small-cell lung cancer. Nature 448: 561-566, 2007.

11. Choi YL, Takeuchi K, Soda M, et al: Identification of novel isoforms of the EML4-ALK transforming gene in non-small cell lung cancer. Cancer Res 68: 4971-4976, 2008.

12. Weiss J, Sos ML, Seidel D, et al: Frequent and focal FGFR1 amplification associates with therapeutically tractable FGFR 1 dependency in squamous cell lung cancer. Sci Transl Med 2: 62ra93, 2010.

13. Costa DB, Halmos B, Kumar A, et al: BIM mediates EGFR tyrosine kinase inhibitor-induced apoptosis in lung cancers with oncogenic EGFR mutations. PLoS Med 4: 1669-1680, 2007.

14. Yasuda H, de Figueiredo-Pontes LL, Kobayashi S and Costa DB: Preclinical rationale for use of the clinically available multitargeted tyrosine kinase inhibitor crizotinib in ROS1-translocated lung cancer. J Thorac Oncol 7: 1086-1090, 2012.

15. Liao RG, Jung J, Tchaicha J, et al: Inhibitor-sensitive FGFR2 and FGFR3 mutations in lung squamous cell carcinoma. Cancer Res 73: 5195-5205, 2013.

16. Dutt A, Ramos AH, Hammerman PS, et al: Inhibitor-sensitive FGFR1 amplification in human non-small cell lung cancer. PLoS One 6: e20351, 2011

17. Pardo OE, Latigo J, Jeffery RE, et al: The fibroblast growth factor receptor inhibitor PD173074 blocks small cell lung cancer growth in vitro and in vivo. Cancer Res 69: 8645-8651, 2009.

18. Dieci MV, Arnedos M, Andre F and Soria JC: Fibroblast growth factor receptor inhibitors as a cancer treatment: from a biologic rationale to medical perspectives. Cancer Discov 3: 264-279, 2013.

19. Turner $\mathrm{N}$ and Grose R: Fibroblast growth factor signalling: from development to cancer. Nat Rev Cancer 10: 116-129, 2010.

20. Donnem T, Al-Shibli K, Al-Saad S, Busund LT and Bremnes RM: Prognostic impact of fibroblast growth factor 2 in non-small cell lung cancer: coexpression with VEGFR-3 and PDGF-B predicts poor survival. J Thorac Oncol 4: 578-585, 2009.

21. Rades D, Seibold ND, Gebhard MP, Noack F, Bruchhage KL and Schild SE: Fibroblast growth factor 2 is of prognostic value for patients with locally advanced squamous cell carcinoma of the head and neck. Strahlenther Onkol 190: 68-74, 2014.

22. Wilson TR, Fridlyand J, Yan Y, et al: Widespread potential for growth-factor-driven resistance to anticancer kinase inhibitors. Nature 487: 505-509, 2012.

23. Nomura S, Yoshitomi H, Takano S, et al: FGF10/FGFR2 signal induces cell migration and invasion in pancreatic cancer. Br J Cancer 99: 305-313, 2008.

24. Ohgino K, Soejima K, Yasuda H, et al: Expression of fibroblast growth factor 9 is associated with poor prognosis in patients with resected non-small cell lung cancer. Lung Cancer 83: 90-96, 2014. 
25. Yin Y, Betsuyaku T, Garbow JR, Miao J, Govindan R and Ornitz DM: Rapid induction of lung adenocarcinoma by fibroblast growth factor 9 signaling through FGF receptor 3. Cancer Res 73: 5730-5741, 2013.

26. Terai H, Soejima K, Yasuda H, et al: Activation of the FGF2FGFR 1 autocrine pathway: a novel mechanism of acquired resistance to gefitinib in NSCLC. Mol Cancer Res 11: 759-767, 2013.
27. Lefevre G, Babchia N, Calipel A, et al: Activation of the FGF2/ FGFR1 autocrine loop for cell proliferation and survival in uveal melanoma cells. Invest Ophthalmol Vis Sci 50: 1047-1057, 2009.

28. Fillmore CM, Gupta PB, Rudnick JA, et al: Estrogen expands breast cancer stem-like cells through paracrine FGF/Tbx3 signaling. Proc Natl Acad Sci USA 107: 21737-21742, 2010. 